\title{
Analysis of holographic reflection gratings recorded in polyvinyl alcohol/acrylamide photopolymer
}

\author{
Elena Fernandez, ${ }^{1,3, *}$ Manuel Perez-Molina, ${ }^{2,3}$ Rosa Fuentes, ${ }^{1,3}$ Manuel Ortuño, ${ }^{2,3}$ \\ Cristian Neipp, ${ }^{2,3}$ Augusto Belendez, ${ }^{2,3}$ and Inmaculada Pascual ${ }^{1,3}$ \\ 'Departamento de Óptica, Farmacología y Anatomía, Universidad de Alicante, Apartado 99, \\ Alicante E-03080, Spain \\ ${ }^{2}$ Departamento de Física, Ingeniería de Sistemas y Teoría de la Señal, Universidad de Alicante, \\ Apartado 99, Alicante E-03080, Spain \\ ${ }^{3}$ Instituto Universitario de Física Aplicada a las Ciencias y las Tecnologías, Universidad de Alicante, \\ Apartado 99, Alicante E-03080, Spain \\ *Corresponding author: elena.fernandez@ua.es
}

Received 11 October 2012; revised 17 January 2013; accepted 25 January 2013; posted 28 January 2013 (Doc. ID 177855); published 5 March 2013

\begin{abstract}
Holographic reflection gratings in a polyvinyl alcohol/acrylamide based photopolymer were stored using symmetrical geometry in three different thicknesses of the material. The advantage of symmetrical geometry is that exact expressions for transmittance, reflectance, and electric fields can be obtained analytically. Using these expressions, experimental data were fitted to obtain parameters such as refractive index modulation, spatial period of the grating, optical thickness or shrinkage of the material. (C) 2013 Optical Society of America
\end{abstract}

OCIS codes: $\quad 090.0090,090.7330,160.5470$.

\section{Introduction}

In the last decade, holography has acquired great importance since devices are being created that can store information throughout the volume of the material thereby increasing the storage capacity in comparison with two-dimensional devices that only store information on the surface. Pioneering companies in this field, such as Bayer Material Science and InPhase, even came together to create the Tapestry [1-4], the first prototype holographic optical storage system that is being used by leading companies and is capable of storing from 200 Gbytes to 1.6 Tbytes in a disk $130 \mathrm{~mm}$ in diameter.

The most widely studied branch of holography, which has given rise to a large number of papers, is transmission holography [5]. However, leading

$1559-128 \mathrm{X} / 13 / 081581-10 \$ 15.00 / 0$

(C) 2013 Optical Society of America companies such as Bayer are beginning to conduct studies aimed at using reflection holography [6]. To obtain more compact systems, it would be interesting to design reflection holographic memories to which the current technology for reading CD's and DVD's designed for reflection holograms may be applied.

In recent years, various studies based on reflection holography have been carried out. In some cases, color reflection holograms have been stored [7], and thanks to their properties, relative humidity sensors based on the color of the hologram have been created [8]. Other studies have examined how certain materials behave when reflection gratings are stored in them [6, $\underline{9}-\underline{12}]$, and multiplexed gratings have even been obtained [13].

Moreover, in the bibliography, there are authors who have recorded reflection holographic gratings in two different ways: (a) with an asymmetric geometry [6] and (b) with a symmetric geometry [14-17]. 
The advantage of a symmetric geometry is that the interference fringes of the grating are parallel to the recording material, in which case it may be assumed that the relative dielectric permittivity of the grating $\varepsilon(z)$ only varies along the $z$ axis (perpendicular to the material surface) [18-20].

In this study, reflection holographic gratings are recorded using a symmetric geometry in a polyvinyl alcohol (PVA)/acrylamide (AA) photopolymer [21,22]. The photopolymer is composed of AA as the polymerizable monomer, triethanolamine (TEA) as radical generator, $\mathrm{N}, \mathrm{N}^{\prime}$-methylene-bis-acrylamide (BMA) as cross-linker, yellowish eosin (YE) as sensitizer and a binder of PVA. The resulting layers are about 40, 70, and $110 \mu \mathrm{m}$ thick.

The gratings stored in each of these layers are reconstructed with a spectrophotometer. To analyze the experimental results, a rigorous electromagnetic model is applied to determine the fields inside the grating as well as its transmittance and reflectance as a function of its dielectric permittivity $\varepsilon(z)$. Since it is assumed that the permittivity exhibits a sinusoidal periodicity, our model enables the exact solution of the Helmholtz equation to be obtained as linear combinations of Mathieu functions [18,20]. The fact that an exact analytical solution is obtained makes it easier to determine characteristic parameters of the grating, such as refractive index modulation, spatial period, or optical thickness of the grating, from the fitting of the model to the experimentally obtained transmittances.

With our exact model, it is possible to analytically compute the electromagnetic fields throughout the grating thickness using Mathieu functions as the exact solution of the Helmholtz equation. Applying diffusion models to analyze hologram formation during exposure is an interesting study that requires determining the exact fields throughout the grating. While this issue has been widely dealt with in transmission holograms, it has not yet been addressed in reflection holograms, for which our exact model holds. Our study thus aims to be the first step in addressing hologram formation in reflection gratings. This requires determining the exact electromagnetic fields inside the material by means of a rigorous model like the one presented in this paper.

\section{Theoretical Model}

A. Analytical Expressions for Reflectance, Transmittance, and Electric Field

As mentioned in the introduction, holographic reflection gratings were recorded in a PVA/AA based photopolymer. For this purpose, two plane waves were made to interfere using a symmetric geometry (the recording beams fall one on each side of the material at the same angle). With this geometry, a periodic structure formed by interference fringes was generated as shown in Fig. 1. In this figure, an incident electromagnetic plane wave $E_{i}$ at an angle $\theta_{i}$ is used to reconstruct a symmetrical reflection grating characterized by a permittivity $\varepsilon(z)$ in $0 \leq z \leq L$, which is surrounded by two semi-infinite media with refractive indexes $n_{1}$ and $n_{3}$. The thickness $L$, in which the reflection grating is stored, is called optical thickness [23]. The incident wave gives rise to a reflected plane wave at an angle $\theta_{r}$ in the region with a refractive index $n_{1}$ and a transmitted plane wave at an angle $\theta_{t}$ in the region with refractive index $n_{3}$.

Our main purpose is to determine the amplitudes of the reflected $\left(E_{r}\right)$ and transmitted $\left(E_{t}\right)$ waves as well as the total electric field $E(z)$ inside the reflection grating $(0<z<L)$. Since photopolymers are nonmagnetic materials $(\mu=1)$, the internal electric field $E(z)$ is obtained by solving the Helmholtz equation and applying adequate boundary conditions at $z=0$ and $z=L$. The main advantage of this procedure is that the reflection grating permittivity has a sinusoidal spatial profile, which makes it possible to obtain an exact analytical solution for the Helmholtz equation, and thus for $E(z), E_{r}$, and $E_{t}$. Consequently, analytical expressions for the transmittance and reflectance of a symmetric grating stored by reflection may be obtained $[18,20]$. This allows us to extract a priori unknown characteristic parameters of the recorded gratings, such as index modulation, $\Delta n$, spatial period, $\Lambda$, or optical thickness $L$, when other recording and reconstruction parameters of the diffraction grating (such as the mean index of the recording material or the recording and reconstruction angles of the grating) are known.

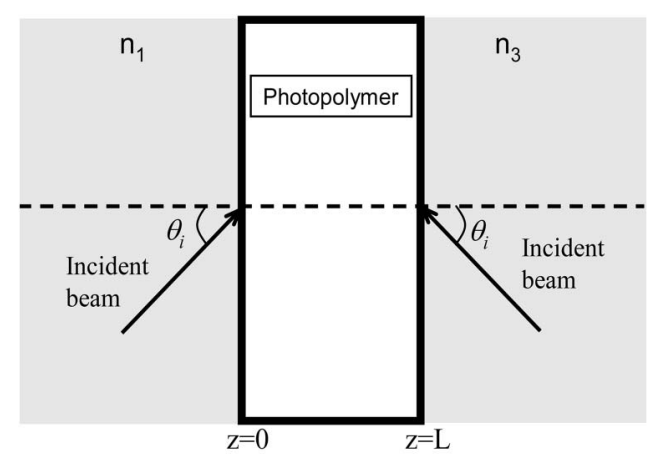

(a)

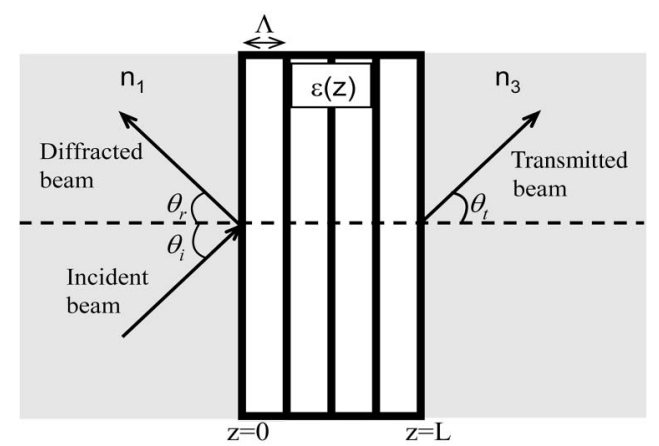

(b)

Fig. 1. Schematic diagram of a holographic reflection grating with symmetric geometry. (a) Recording stage with two beams at the same incidence angle. (b) Reconstruction stage. 
In the diagram in Fig. 1, the generalized Snell's law [24] states that

$$
\theta_{i}=\theta_{r} \quad n_{1} \cdot \sin \theta_{i}=n_{3} \cdot \sin \theta_{t} .
$$

In our analysis, the reconstruction angle $\theta_{i}=0^{\circ}$ (incidence is normal), so the behavior of the TE and TM modes is essentially the same and thus it is sufficient to analyze the TE mode. On the other hand, the boundary conditions imply continuity for $E(z)$ and its derivative $\partial E / \partial z$ at $z=0$ and $z=L$. Denoting the amplitude reflection and transmission coefficients by $r=E_{r} / E_{i}$ and $t=E_{t} / E_{i}$, respectively, the Helmholtz equation (that describes the behavior of the electric field) and the boundary conditions can be written as

$$
\begin{aligned}
& E^{\prime \prime}(z)+k_{0}^{2} \varepsilon(z) E(z)=0 \\
& E(0)=(1+r) E_{i}, \quad E(L)=t \cdot E_{i} \\
& E^{\prime}(0)=i k_{0} n_{1}(1-r) E_{i}, \quad E^{\prime}(L)=i k_{0} n_{3} t E_{i},
\end{aligned}
$$

where $k_{0}=2 \pi / \lambda$ is the vacuum wavenumber, $\lambda$ is the vacuum wavelength of the electromagnetic fields, and the prime denotes derivatives with respect to $z$. In Eq. (2), it should be noted that $r$ and $t$ are $a$ priori unknowns that appear in the boundary conditions, which in turn are necessary to obtain $E(z)$. To overcome this difficulty, we consider the spatial inversion $z \rightarrow(L-z)$, which transforms the previous equations into the initial value problem given by

$$
\begin{aligned}
& E_{p}^{\prime \prime}(z)+k_{0}^{2} \varepsilon(L-z) E_{p}(z)=0 \\
& E_{p}(0)=1, \quad E_{p}^{\prime}(0)=-i k_{0} n_{3} .
\end{aligned}
$$

Following the procedure described in [25], the solution $E_{p}(z)$ of Eq. (3) allows the amplitude reflection and transmission coefficients $r$ and $t$, as well as the electric field amplitude $E(z)$, inside the material to be obtained as

$r=\frac{i k_{0} n_{1} E_{p}(L)+E_{p}^{\prime}(L)}{i k_{0} n_{1} E_{p}(L)-E_{p}^{\prime}(L)}, \quad t=\frac{2 i k_{0} n_{1}}{i k_{0} n_{1} E_{p}(L)-E_{p}^{\prime}(L)}$,

$$
E(z)=t E_{i} E_{p}(L-z), \quad 0 \leq z \leq L,
$$

whereas the electric field in the regions that surround the reflection grating can be obtained as a function of the incident field $E_{i}$ and the coefficients $r$ and $t$ as
$E(z)=\left\{\begin{array}{l}E_{i} \exp \left(i k_{0} n_{1} z\right)+r E_{i} \exp \left(-i k_{0} n_{1} z\right) \quad \text { if } z<0 \\ t \cdot E_{i} \exp \left(i k_{0} n_{3}(z-L)\right) \quad \text { if } z>L\end{array}\right.$.

From Eq. (4), the power reflectance $R$ and transmittance $T$ coefficients for the TE mode can be obtained as

$$
R=|r|^{2}, \quad T=\frac{\operatorname{Re}\left[n_{3}\right]}{\operatorname{Re}\left[n_{1}\right]}|t|^{2},
$$

where Re denotes the real part.

In the case under study, i.e., symmetric holographic reflection gratings, the permittivity profile $\varepsilon(z)$ that best describes the properties of this type of grating can be written as

$$
\varepsilon(z)=\varepsilon_{0}+\varepsilon_{m} \cos \left(\frac{2 \pi z}{\Lambda}\right), \quad 0 \leq z \leq L,
$$

where $\varepsilon_{0}$ is the average permittivity and $\varepsilon_{m}$ is the permittivity modulation. To obtain $E_{p}(z)$ for the reflection grating considered, Eq. (8) is substituted into Eq. ( ) to give

$$
E_{p}^{\prime \prime}(z)+k_{0}^{2}\left(\varepsilon_{0}+\varepsilon_{m} \cos \left(\frac{2 \pi(L-z)}{\Lambda}\right)\right) E_{p}(z)=0 .
$$

Equation (9) is known as Mathieu's differential equation and has an exact solution that can be obtained as a linear combination of Mathieu functions [26]:

$E_{p}(z)=C_{1} m c\left(a, q, \frac{\pi(L-z)}{\Lambda}\right)+C_{2} m s\left(a, q, \frac{\pi(L-z)}{\Lambda}\right)$,

where $m c$ and $m s$ are the even and odd Mathieu functions, respectively, $a$ and $q$ are expressed as:

$$
a=\frac{4 \varepsilon_{0} \Lambda^{2}}{\lambda^{2}}, \quad q=-\frac{2 \varepsilon_{m} \Lambda^{2}}{\lambda^{2}}
$$

and $C_{1}$ and $C_{2}$ are integration constants (a priori unknown). Combining Eq. (10) with the boundary conditions $E_{p}(0)=1$ and $E_{p}^{\prime} \overline{(0)}=-i k_{0} n_{3}$, we obtain a linear system of two equations with two unknowns $C_{1}$ and $C_{2}$, whose solution can be expressed as

$$
C_{1}=\frac{\pi \cdot \mathrm{ms}^{\prime}\left(a, q, \frac{\pi L}{\Lambda}\right)-i k_{0} n_{3} \Lambda \cdot m s\left(a, q, \frac{\pi L}{\Lambda}\right)}{\pi \cdot\left(\mathrm{mc}\left(a, q, \frac{\pi L}{\Lambda}\right) \cdot \mathrm{ms}^{\prime}\left(a, q, \frac{\pi L}{\Lambda}\right)-\mathrm{ms}\left(a, q, \frac{\pi L}{\Lambda}\right) \cdot \mathrm{mc}^{\prime}\left(a, q, \frac{\pi L}{\Lambda}\right)\right)},
$$




$$
C_{2}=\frac{i k_{0} n_{3} \Lambda \cdot \mathrm{mc}\left(a, q, \frac{\pi L}{\Lambda}\right)-\pi \cdot \mathrm{mc}^{\prime}\left(a, q, \frac{\pi L}{\Lambda}\right)}{\pi \cdot\left(\mathrm{mc}\left(a, q, \frac{\pi L}{\Lambda}\right) \cdot \mathrm{ms}^{\prime}\left(a, q, \frac{\pi L}{\Lambda}\right)-\mathrm{ms}\left(a, q, \frac{\pi L}{\Lambda}\right) \cdot \mathrm{mc}^{\prime}\left(a, q, \frac{\pi L}{\Lambda}\right)\right)},
$$

where $\mathrm{mc}^{\prime}$ and $\mathrm{ms}^{\prime}$, respectively, denote the derivative of the even and odd Mathieu functions with respect to the variable $z$. Equations (10)-(12) provide the analytical function $E_{p}(z)$, which can be substituted into Eqs. ( $\underline{4})$ and (ㅁ) to obtain exact analytical expressions for the reflection and transmission coefficients $t$ and $r$ [and the power coefficients $R$ and $T$ from Eq. (7)] and for the electric field $E(z)$. In particular, assuming the typical condition $n_{1}=$ $n_{0}=\sqrt{\varepsilon_{0}}=n_{3}$ of index matching between the grating and the adjacent regions, Eqs. (4) and (7) allow an exact analytical expression for the power transmission coefficient to be obtained as a function of $\lambda, \Lambda, L, \varepsilon_{0}$, and $\varepsilon_{m}$, i.e.,

$$
T\left(\lambda, \Lambda, L, \varepsilon_{0}, \varepsilon_{m}\right)=\frac{4 k_{0}^{2} n_{0}^{2}}{\left|i k_{0} n_{0} E_{p}(L)-E_{p}^{\prime}(L)\right|^{2}},
$$

where $E_{p}(L)$ depends on $\lambda, \Lambda$, and $\varepsilon_{m}$ through the previously defined parameters $a, q, C_{1}$, and $C_{2}$. It should be remembered that the above (exact) analytical expressions for the grating transmittance and fields are obtained assuming the relative permittivity $\varepsilon(z)$ has the sinusoidal profile given in Eq. (8). Although it would be possible to research the grating behavior assuming nonsinusoidal profiles for $\varepsilon(z)$, this would probably lead to a Helmholtz equation, Eq. (3), without an exact elementary analytical solution unsuitable for fitting the experimental transmittance as a function of $\lambda$.

Moreover, it should be noted that the method developed in this paper allows the exact transmittance to be obtained directly as a function of the wavelength $\lambda$ without involving any transfer matrix formalism. Consequently, in comparison with the general transfer matrix method reported in [18,20,25] (which is strictly numerical without analytical expressions), our method is more efficient for fitting experimental transmittances of sinusoidal reflection gratings and obtaining their characteristic parameters (as will be shown in Section $\underline{4}$ ).

\section{B. Comparison with Finite-Difference Time-Domain Simulation Results}

To demonstrate the accurateness and advantages of our analytical model, we compare it with finitedifference time-domain (FDTD) simulations. For this purpose, we consider a reflection grating with the geometry depicted in Fig. 1 assuming the following parameters:

$\varepsilon_{0}=2.292, \quad \varepsilon_{m}=0.07, \quad \Lambda=0.1965 \mu \mathrm{m}$,

$L=20 \mu \mathrm{m}, \quad n_{1}=n_{3}=\sqrt{\varepsilon_{0}}=1.514$.
To carry out FDTD simulation of the reflection grating defined by Eqs. (8) and (14), we consider a one-dimensional computational window with a lattice increment $\Delta z=5.95 \mathrm{~nm}$ placed between the boundary planes $z=-50 \mu \mathrm{m}$ and $z=50 \mu \mathrm{m}$, which are indicated by vertical red dotted lines in Fig. 2 . To avoid reflections at these boundary planes, we add two auxiliary perfectly matched layer (PML) regions of $20 \mu \mathrm{m}$ thicknesses surrounding the computational window at each of its sides, in which the absorbed electric field is not strictly regarded as a simulation result (we focus on the computational window $-50 \mu \mathrm{m} \leq z \leq 50 \mu \mathrm{m})$.

To obtain the frequency-domain incident electric field $E_{i}(\lambda)$ on the reflection grating at $z=0$ we assume a constant relative permittivity $\varepsilon_{0}=2.292$ throughout the computational window and a free current pulse at $z=-40 \mu \mathrm{m}$ as an excitation source that creates two identical counter-propagating light pulses. As shown in Fig. 2(a) (Media 1), the pulse propagating toward $z=-\infty$ is absorbed in the left PML and does not reach the plane $z=0$, so $E_{i}(\lambda)$

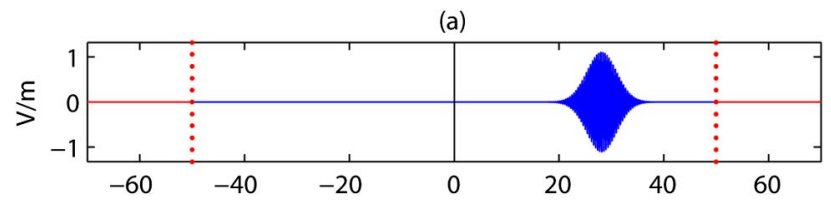

(b)

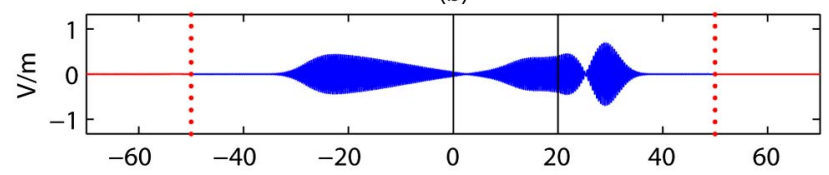

(c)

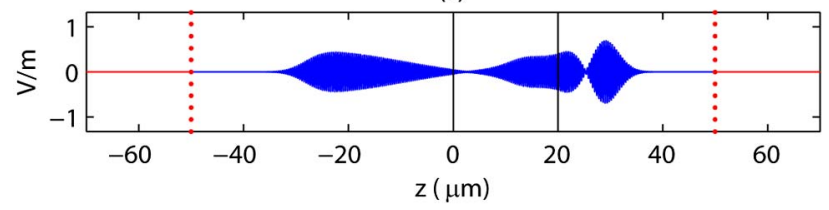

Fig. 2. (Color online) Movie of the time-domain electric field in the schematic diagram in Fig. 1 computed between the planes $z=$ $-50 \mu \mathrm{m}$ and $z=50 \mu \mathrm{m}$, which separate the computational region from the PMLs and are indicated by vertical red dotted lines (Media 1). (a) Incident field propagation in a homogeneous medium $\left(\varepsilon_{0}=2.292\right)$ obtained via FDTD. The vertical black continuous line indicates the plane $z=0$. (b) Electric field propagation obtained via FDTD for the reflection grating defined by Eqs. (8) and (14). The vertical black continuous lines indicate the grating boundaries at $z=0$ and $z=20 \mu \mathrm{m}$. (c) Electric field propagation for the same reflection grating obtained through the inverse Fourier transform of the electric field given by Eqs. (5) and (6). In this case, the electric fields are not considered outside the computational region, i.e., inside the PML regions, where they are plotted as null values (red color). 
is obtained as the Fourier transform of the timedomain electric field at $z=0$ (which is only due to the pulse propagating toward $z=+\infty$ ). In the second step of our FDTD analysis, we consider the same excitation source but now include the grating permittivity [Eqs. (8) and (14)] between the planes $z=0$ and $z=20 \mu \overline{\mathrm{m}}$, which are indicated by vertical black continuous lines in Fig. 2(b). The temporal evolution of the electric field depicted in Fig. 2(b) (Media 1) shows multiple Bragg reflections inside the grating that give rise to a considerable temporal spread of the fields.

Once $E_{i}(\lambda)$ has been computed, Eqs. (10)-(12) and (4)-(7) provide exact analytical expressions for the frequency-domain electric field along the $z$ axis. Consequently, the inverse Fourier transform of this analytical field with respect to $\omega=2 \pi c / \lambda$ ( $c$ is the speed of light in the vacuum) gives the electric field in the computational window as a function of time, which is depicted in Fig. 2(c) (Media 1). As can be seen, the FDTD results depicted in Fig. 2(b) (Media 1) coincides exactly with the time-domain fields obtained with our analytical model in Fig. 2(c) (Media 1) in the computational region $-50 \mu \mathrm{m} \leq z \leq$ $50 \mu \mathrm{m}$ as long as the counter-propagating pulse in Fig. 2(b) is absorbed in the left PML. In this sense, in Eq. (6) of our model, the incident field is regarded as a nonmonochromatic plane wave that propagates from $z=-\infty$ to $z=0$. The excitation source that generates the pulse in the FDTD scheme is thus disregarded, which explains the absence of the pulse propagating toward $z=-\infty$ in Fig. 2(c). Apart from this minor difference between Figs. 2(b) and 2(c) (Media 1), the FDTD results coincide exactly with the fields obtained using our analytical model, which corroborates its accurateness.

In Section 4, we use the theoretical power transmittance coefficient $T\left(\lambda, \Lambda, L, \varepsilon_{0}, \varepsilon_{m}\right)$ of Eq. (13) to fit the experimental results to obtain the parameters of the grating.

\section{Experimental}

\section{A. Preparation of the Material}

The holograms are recorded in a photopolymer composed of AA as a monomer, TEA as a radical generator, BMA as a cross-linker, YE as a sensitizer and a binder of PVA.

The main advantages and benefits of choosing PVA/AA are the following: (i) it is easy and inexpensive to prepare in a conventional laboratory and can be reconstructed without postprocessing, (ii) it has a high sensitivity, (iii) it is suitable for making layers with a wide range of thicknesses $(40-1000 \mu \mathrm{m})$, (iv) its behavior can be modified by means of simple modifications of the composition and processing stepsin particular, its spectral sensitivity can be tuned by changing the dye, and (v) the components of the standard formulation can be substituted to give a low level of toxicity.
Table 1. Concentrations of the Photopolymer Composition

\begin{tabular}{lc}
\hline & Composition \\
\hline Polyvinyl alcohol (PVA) & $8.3 \% \mathrm{~m} / \mathrm{v}$ \\
Acrylamide (AA) & $0.33 \mathrm{M}$ \\
Triethanolamine (TEA) & $0.19 \mathrm{M}$ \\
Yellowish eosin (YE) & $2.45 \cdot 10^{-4} \mathrm{M}$ \\
$\mathrm{N}, \mathrm{N}^{\prime}$ methylene-bis-acrylamide (BMA) & $0.027 \mathrm{M}$ \\
\hline
\end{tabular}

Table 1 shows the component concentrations of the photopolymer composition used to obtain layers of 40, 70, and $110 \mu \mathrm{m}$ in thickness.

A solution of PVA in water forms the matrix and this is used to prepare the mixture of AA, BMA, and photopolymerization initiator system composed of TEA and YE. The mixture is made under red light, deposited by gravity on a $22 \mathrm{~cm} \times 40 \mathrm{~cm}$ glass plate, and left in the dark for approximately one day to allow the water to evaporate in conditions of temperature, $T$, between $18^{\circ} \mathrm{C}$ and $20^{\circ} \mathrm{C}$, and relative humidity, $30 \%-40 \%$. Once dry, the glass is cut into squares of $6 \mathrm{~cm} \times 6 \mathrm{~cm}$.

The refractive index of the material is calculated using the method described in [27]. In this method, the experimental reflectance of $p$-polarized light is measured as a function of the incident angle, and the value of the refractive index of the layer is obtained using the theoretical equation for reflectance. Fitting the theoretical reflectance to the experimental data, a value for the refractive index of $n_{0}=1.514$ was obtained.

Figure 3 shows the transmittance of the material versus wavelength for the three thicknesses. As can be seen, the three plots have the same shape with the minimum transmittance (maximum absorption) at the recording wavelength $\lambda=532 \mathrm{~nm}$. Consequently, the material composition is optimized so that the material is as sensitive as possible at the recording wavelength. The only difference between the three plots is that the transmittance decreases when the layer thickness increases.

In the range between $\lambda=570 \mathrm{~nm}$ and $\lambda=590 \mathrm{~nm}$, the transmittance is maximum and thus the material does not absorb or absorbs very little.

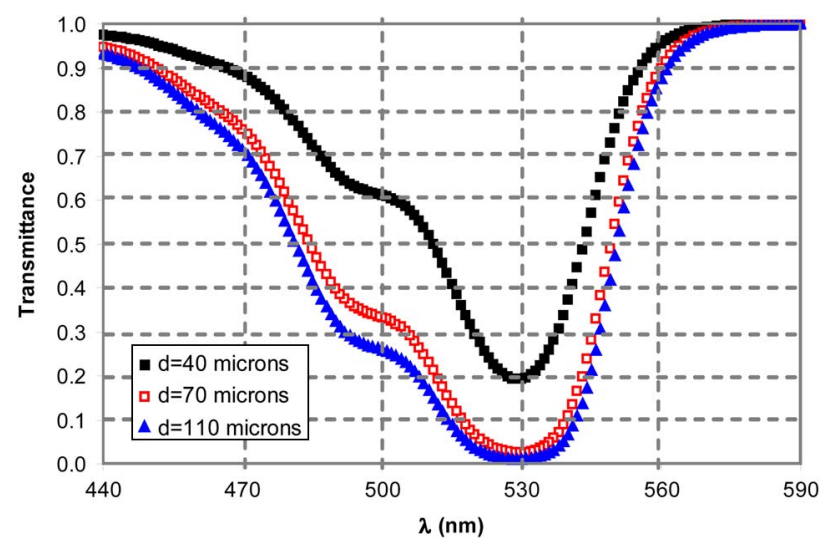

Fig. 3. (Color online) Transmittance of the unexposed photopolymer plate. 
The geometries for the grating recording and reconstruction were chosen so that the Bragg wavelength would appear in this nonabsorbing spectral range (see Section 3B) in order to avoid overlapping of the Bragg and absorption peaks (such overlapping would probably mask the results).

\section{B. Holographic Setup}

Reflection holographic gratings were stored using a Nd:YV04 laser (Coherent Verdi V2) with a wavelength of $532 \mathrm{~nm}$, to which the material was sensitive. A symmetric geometry was used to obtain gratings with interference fringes parallel to the recording material as shown in Fig. 1. The setup is shown in Fig. 4 . The polarized beam emitted by the laser was split into two beams with a beam splitter. Each beam was expanded and filtered using a microscope objective and a pinhole. Then the beams passed through a series of lenses and diaphragms to obtain collimated beams with the desired diameter. The total intensity of the recording beams was $30 \mathrm{~mW} / \mathrm{cm}^{2}$ with an intensity ratio of $1: 1$. The two laser beams were spatially overlapped at the recording medium intersecting at an angle of $45^{\circ}$ (measured in air).

Using the value of the refractive index of the material obtained in the previous section (we disregarded the small change that took place during recording as it was smaller than the refractive index modulation and thus did not affect the results), the intersection angle in air and the recording wavelength, the theoretical spatial period of the grating, $\Lambda_{\text {th }}$, was $0.1987 \mu \mathrm{m}$ applying Eq. (15) (therefore, the spatial frequency of the recorded reflection gratings was 5032 lines/mm):

$$
\Lambda=\frac{\lambda}{2 \sqrt{n_{0}^{2}-\sin ^{2} \theta}} .
$$

During the photopolymerization process, the conversion of monomer molecules into a polymer network is accompanied by close packing of the growing chains and a subsequent reduction in volume. This phenomenon, known as photopolymerization shrinkage, is particularly relevant in the case of reflection gratings and occurs when the effective period of the grating recorded in the sample, $\Lambda_{\text {exp }}$, is less than that defined by the geometrical conditions of the recording, $\Lambda_{\mathrm{th}}$. Shrinkage of the material induces a change in the fringe spacing $\Delta \Lambda=$ $\Lambda_{\text {th }}-\Lambda_{\text {exp }}$. The ultimate effect is a shift in the wavelength at which the grating peak appears. So, we can define a parameter called optical shrinkage [28] as

$$
S_{\mathrm{opt}}=\frac{\lambda_{\mathrm{th}}-\lambda_{\mathrm{exp}}}{\lambda_{\mathrm{th}}}
$$

where $\lambda_{\text {th }}$ is the theoretical wavelength where the grating peak should appear if there was no shrinkage and $\lambda_{\exp }$ is the experimental wavelength where the grating peak actually appears. Substituting $n_{0}=$ 1.514, $\Lambda=\Lambda_{\text {th }}=0.1987 \mu \mathrm{m}$ and $\theta=\theta_{i}=0^{\circ}$ into Eq. (15), we obtain a theoretical wavelength $\lambda_{\text {th }}=\overline{602} \mathrm{~nm}$.

The optical shrinkage of the gratings was calculated for each sample thickness, as discussed in Section $\underline{4}$.

\section{Results and Discussion}

In this section, the results obtained recording holographic reflection gratings in a PVA/AA based photopolymer with a symmetric geometry are presented. The gratings were stored in three different sample thicknesses $(40,70$, and $110 \mu \mathrm{m})$ to determine which thickness is the most appropriate for storing reflection gratings with the highest refractive index modulation. In Fig. $\underline{3}$, the grating reconstruction was carried out in a spectral range $(585-605 \mathrm{~nm})$ where absorption is negligible, so the conservation of energy principle implies that the transmittance $T(\lambda)$ and reflectance $R(\lambda)$ are approximately complementary, i.e., $T(\lambda)+R(\lambda) \approx 1$ and thus $R(\lambda) \approx 1-T(\lambda)$. Consequently, analyzing just the transmittance $T(\lambda)$ will suffice.

Once the transmittance of each grating was measured as a function of wavelength, the corresponding theoretical expression obtained in Eq. (13) was fitted to the experimental transmittance in two steps. First, $T\left(\lambda, \Lambda, L, \varepsilon_{0}, \varepsilon_{m}\right)$ was obtained as an analytical function of $\lambda, \Lambda, L$, and $\varepsilon_{m}$ for a fixed value $\varepsilon_{0}=n_{0}^{2}$,

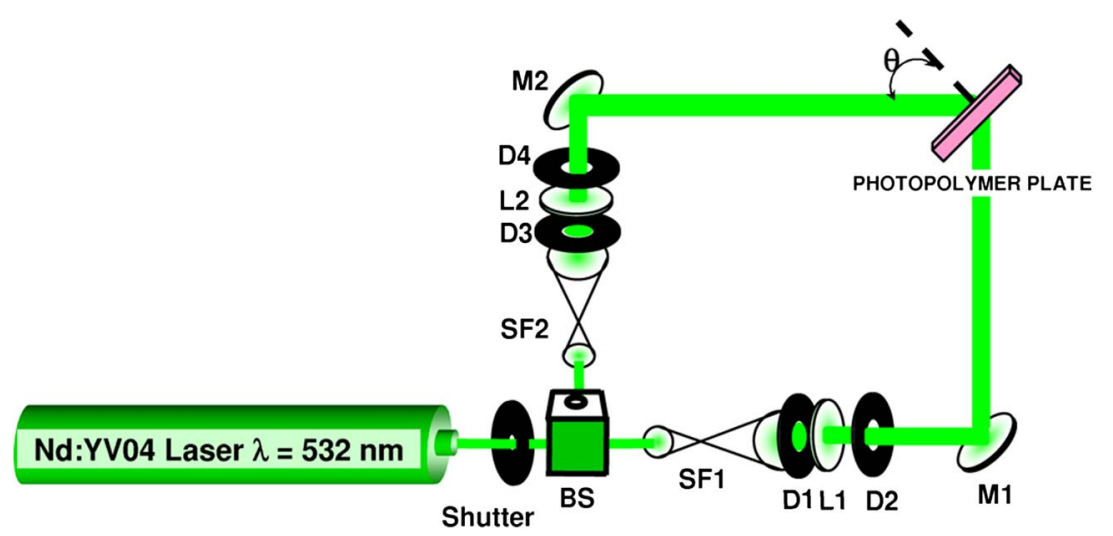

Fig. 4. (Color online) Experimental setup: BS, beam splitter; Mi, mirror; Li, lens; Di, diaphragm; SFi, microscope objective lens and pinhole. 
where $n_{0}$ is the measured average refractive index of the grating (see Section 3A). Second, this analytical transmittance coefficient was fitted to the experimental transmittance using $\Lambda, L$, and $\varepsilon_{m}$ as free parameters to be adjusted by the gradient method to minimize the mean square error (MSE) between the experimental and the analytical transmittance (for each measured $\lambda$ ). The fitting was carried out using sixteen wavelengths between 585 and $600 \mathrm{~nm}$, i.e., the wavelength sample rate was $1 \mathrm{~nm}$, which is sufficiently greater than the spectrophotometer resolution given as $0.1 \mathrm{~nm}$. The MSE obtained for each of the fittings confirmed their good quality. As a result of the fitting, we obtained the experimental grating period $\Lambda$, optical thickness $L$, and grating permittivity modulation $\varepsilon_{m}$, which in turn allowed the experimental wavelength $\lambda_{\exp }=2 n_{0} \Lambda_{\exp }$ [taking $\theta=0$ in Eq. (15)] and refractive index modulation of the grating $\varepsilon_{m} / 2 n_{0}$ to be obtained. Based on these values, it was possible to estimate the shrinkage [28].

Holographic reflection gratings with thicknesses of 40,70 , and $110 \mu \mathrm{m}$ were recorded in a material with the composition shown in Table 1 . They were recorded following the scheme shown in Fig. 4, in which the different exposures corresponded to a constant exposure intensity of $30 \mathrm{~mW} / \mathrm{cm}^{2}$ for different exposure times. Once the gratings were recorded, they were reconstructed measuring the transmittance of the grating as a function of the wavelength using a double-beam spectrophotometer. The plate was placed perpendicular to the beam $\left(\theta=0^{\circ}\right)$. The theoretical transmittance obtained from Eq. (13) was fitted to the experimental transmittance obtained for each grating.

Figure 5 shows the refractive index modulation and the optical thickness as a function of exposure for three different sample thicknesses: $40 \mu \mathrm{m}$ in Fig. 5(a), $70 \mu \mathrm{m}$ in Fig. 5(b), and $110 \mu \mathrm{m}$ in Fig. 5(c). For each sample thickness, the refractive index modulations and optical thicknesses were obtained from the fitting of the transmittance coefficients, which were measured for the different gratings recorded with their corresponding exposures. The MSE obtained for each of the fittings in Figs. 5(a) $-5(\mathrm{c})$ was between $10^{-4}$ and $10^{-6}$, thus confirming the good quality of the fittings.

For the three sample thicknesses considered, the refractive index modulation $\Delta n$ showed a clear tendency to increase as exposure increased, until it reached an approximately constant value with small fluctuations. On the other hand, the optical thickness $L$ showed a clear tendency to decrease as exposure increased, until it reached an approximately constant value with small fluctuations. The small fluctuations in $L$ and $\Delta n$ can be explained taking into account that the process of grating recording (with laser) and reconstruction (with spectrophotometer) was not carried out in real-time, so a different grating was recorded and reconstructed for each different exposure. In addition, one key parameter, which is directly related to the grating diffraction efficiency

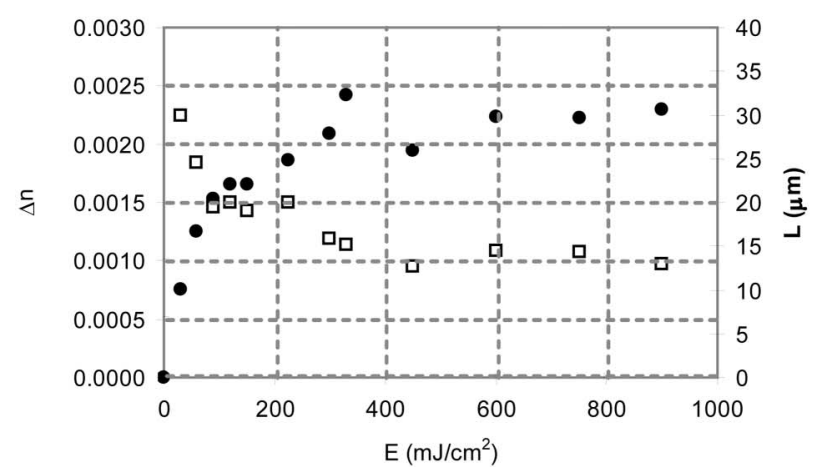

(a)

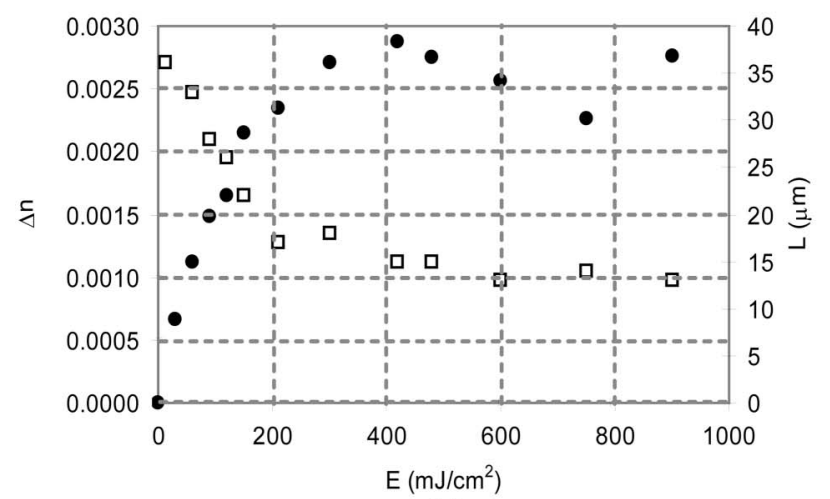

(b)

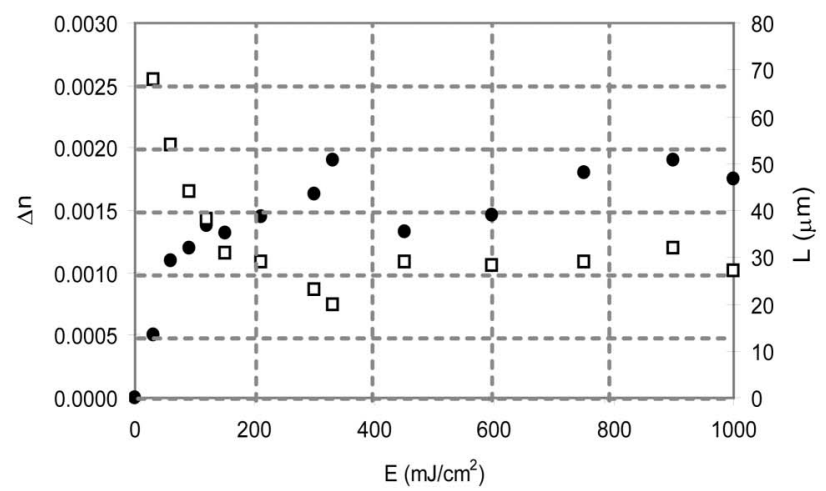

(c)

Fig. 5. Refractive index modulation (solid circles) and optical thickness (empty squares) versus exposure for a material with a sample thickness of (a) 40, (b) 70, and (c) $110 \mu \mathrm{m}$.

for the data depicted in Fig. 5 , is the product $\Delta n \cdot L$ (i.e., refractive index modulation multiplied by optical thickness). In fact, the greater the product $\Delta n \cdot L$ is greater the grating diffraction efficiency is, and vice versa.

For the gratings with a sample thickness of $40 \mu \mathrm{m}$, Fig. 5(a) shows that the refractive index modulation and optical thickness reached approximately constant values of 0.0024 and $15 \mu \mathrm{m}$, respectively, for exposures above $300 \mathrm{~mJ} / \mathrm{cm}^{2}$. On the other hand, the product $\Delta n \cdot L$ was between 0.026 and $0.033 \mu \mathrm{m}$ for exposures above $300 \mathrm{~mJ} / \mathrm{cm}^{2}$.

For a sample thickness of $70 \mu \mathrm{m}$, it can be seen in Fig. 5(b) that the refractive index modulation and optical thickness reached approximately constant values of 0.0026 and $15 \mu \mathrm{m}$, respectively for 
exposures above $300 \mathrm{~mJ} / \mathrm{cm}^{2}$. In this case, the product $\Delta n \cdot L$ was between 0.036 and $0.043 \mu \mathrm{m}$ for exposures above $400 \mathrm{~mJ} / \mathrm{cm}^{2}$.

Finally, for a sample thickness of $110 \mu \mathrm{m}$, Fig. 5(c) shows that the refractive index modulation varied between 0.0013 and 0.0019 , while the optical thickness had an approximately constant value of $28 \mu \mathrm{m}$ for exposures above $300 \mathrm{~mJ} / \mathrm{cm}^{2}$. In this case, the product $\Delta n \cdot L$ was between 0.038 and 0.053 for exposures above $300 \mathrm{~mJ} / \mathrm{cm}^{2}$.

According to these results, the product $\Delta n \cdot L$, and in turn the grating diffraction efficiency, for each sample thickness is approximately constant except for small fluctuations. This explains the opposing tendencies of $\Delta n$ and $L$. When one tends to increase, the other tends to decrease so that the product $\Delta n \cdot L$ remains approximately constant (with some fluctuations).

Another aspect that can be deduced from Figs. 5(a) -5 (c) is the behavior of the optical thickness for the three sample thicknesses considered. The optical thickness for a sample thickness of $70 \mu \mathrm{m}$ is of the same order than for a sample thickness of $40 \mu \mathrm{m}$ for all the exposures considered, as can be seen from a comparison of Figs. 5(a) and 5(b). On the other hand, the optical thickness for a sample thickness of $110 \mu \mathrm{m}$ is considerably higher (nearly double) than for a sample thickness of $70 \mu \mathrm{m}$ for all the exposures considered. These results indicate that optical thickness increases with sample thickness, although not linearly.

The behavior of the refractive index modulation $\Delta n$ for the different sample thicknesses, Figs. 5(a)-5(c) show that the maximum $\Delta n$ for a sample thickness of $70 \mu \mathrm{m}(0.0029)$, is greater than for a thickness of $40 \mu \mathrm{m}(0.0023)$ and $110 \mu \mathrm{m}(0.0019)$. This behavior can be explained by taking into account the absorption and thickness during grating recording. On one hand, the optical thickness tends to increase with sample thickness because interference can occur in a greater thickness and thus more grating planes can be formed. But on the other hand, absorption also increases with sample thickness and limits the interference between the two beams and thus diffraction efficiency. A combination of these two factors explains why: (i) the optical thickness increases with sample thickness but not linearly [23] and (ii) the diffraction efficiency, and in turn the product $\Delta n \cdot L$, also tends to increase slightly with sample thickness [as may be seen in Figs. 6(a)-6(c)] but not linearly. Consequently, when the sample thickness changes from 40 to $70 \mu \mathrm{m}$, the optical thickness remains approximately constant but $\Delta n \cdot L$ increases, so $\Delta n$ also increases. However, when the sample thickness changes from 70 to $110 \mu \mathrm{m}$, the optical thickness increment is more significant than the increment in $\Delta n \cdot L$, so $\Delta n$ decreases.

Figure 6 shows the measured transmittances as a function of wavelength $\lambda$ and the theoretical fit of such transmittances for the reflection gratings with the greatest refractive index modulation $\Delta n$ in each

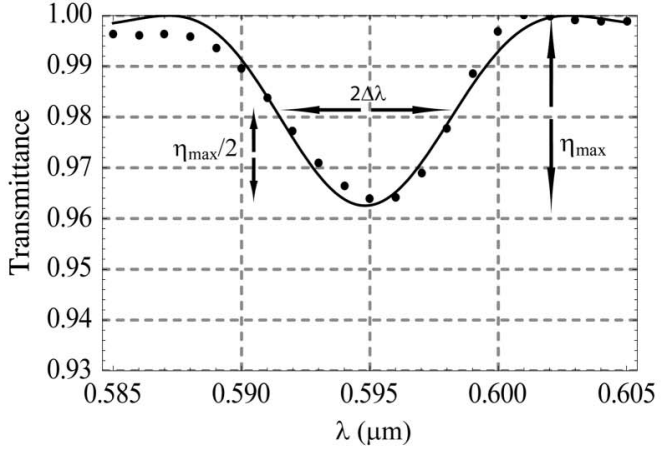

(a)

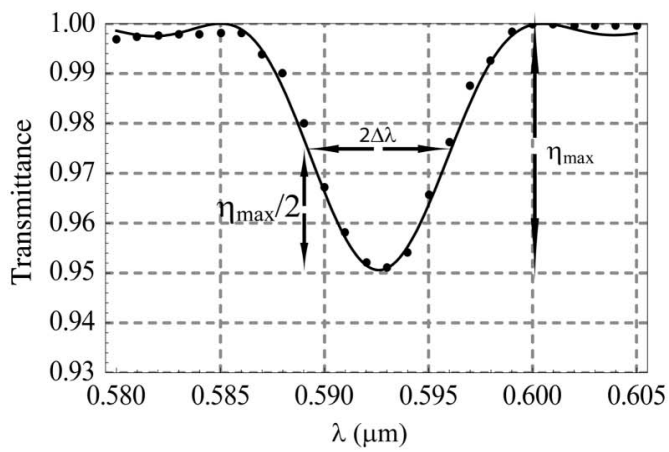

(b)

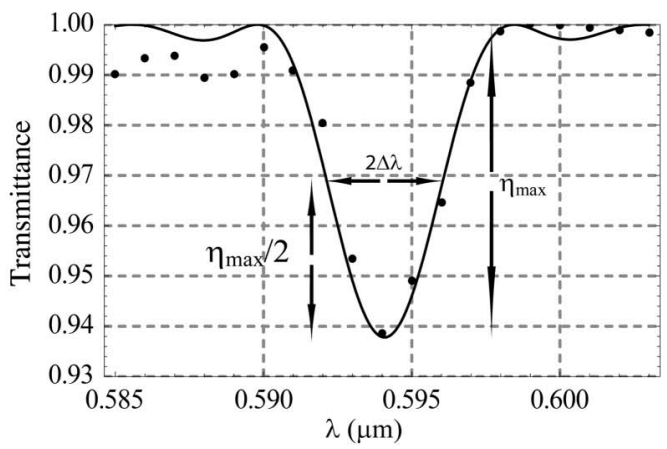

(c)

Fig. 6. Circles represent the experimental transmittance and the continuous line, the theoretical fit of the transmittance for a holographic reflection grating with a sample thickness of (a) 40 , (b) 70 , and (c) $110 \mu \mathrm{m}$.

of the three sample thicknesses considered: $40 \mu \mathrm{m}$ in Fig. 6(a), $70 \mu \mathrm{m}$ in Fig. 6(b), and $110 \mu \mathrm{m}$ in Fig. 6(c). In each case, the continuous line denotes the theoretical transmittance obtained after the fitting to the experimental data corresponding to the peak of the grating, i.e., between 586 and $600 \mathrm{~nm}$.

Figure 6(a) shows the transmittance of the grating recorded at an exposure of $330 \mathrm{~mJ} / \mathrm{cm}^{2}$, whose refractive index modulation $\Delta n=0.0024$ is the greatest of the gratings recorded in a sample thickness of $40 \mu \mathrm{m}$. In this case an MSE of $6.25 \cdot 10^{-6}$ was obtained, which corroborates the good quality of the fitting in accordance with Fig. 6(a). The fitting also provided an experimental spatial period $\Lambda_{\exp }=0.1965 \mu \mathrm{m}$ and an optical thickness $L=15 \mu \mathrm{m}$. The grating peak appears at the Bragg wavelength $\lambda_{\text {exp }}=595 \mathrm{~nm}$ (wavelength that provides maximum reflectance of 
the diffraction grating). Since the previously obtained theoretical wavelength was $\lambda_{\text {th }}=602 \mathrm{~nm}$ (Section 3B), Eq. (16) provides an optical shrinkage $S_{\text {opt }}=1 . \overline{16 \%}$. The optical shrinkage obtained for the gratings with a sample thickness of $40 \mu \mathrm{m}$ varied between $0.8 \%$ and $1.3 \%$.

Figure 6(b) represents the experimental transmittance of the grating with the highest refractive index modulation in Fig. 5(b), which was obtained at an exposure $E=425 \mathrm{~mJ} / \mathrm{cm}^{2}$ and the fit to these experimental data. In this case, an MSE of $3 \cdot 10^{-6}$ was obtained, whereas the results obtained from the fitting were $\Delta n=0.0028, \Lambda_{\exp }=0.1961 \mu \mathrm{m}$, and $L=15 \mu \mathrm{m}$. Since the grating peak appears at $\lambda_{\exp }=$ $593 \mathrm{~nm}$, the optical shrinkage calculated using Eq. (16) is $S_{\text {opt }}=1.5 \%$ in this case, which is the maximum shrinkage obtained for the reflection gratings with a sample thickness of $70 \mu \mathrm{m}$. The optical shrinkage obtained for the gratings with a sample thickness of $70 \mu \mathrm{m}$ varied between $1.1 \%$ and $1.5 \%$.

Finally, Fig. 6(c) depicts the experimental transmittance of the grating with the highest refractive index modulation in Fig. 5(c), which was obtained with an exposure $E=900 \mathrm{~mJ} / \mathrm{cm}^{2}$. The continuous line shows the fit to these experimental data, for which the MSE was $10^{-4}$. The data obtained from the fitting were: $\Delta n=0.0019, \Lambda_{\exp }=0.1962 \mu \mathrm{m}$, and $L=32 \mu \mathrm{m}$. The grating peak appears at $\lambda_{\exp }=$ $594 \mathrm{~nm}$, so the optical shrinkage calculated with Eq. (16) is $S_{\text {opt }}=1.3 \%$. For a sample thickness of $110 \mu \mathrm{m}$, the optical shrinkage varied between $1.1 \%$ and $2.2 \%$. In addition, it can be observed that the transmittance in the spectral range below the Bragg wavelength is slightly smaller than the transmittance in the range above the Bragg wavelength. Such asymmetry is due to a slight absorption (less than $1 \%$ ) that does not affect the results for the main transmittance peak.

\section{Conclusion}

In this paper, holographic reflection gratings were stored in a PVA/AA based photopolymer of three different thicknesses. A symmetrical geometry was used in which the recording beams interfere in the material at the same angle, thus making it possible to record gratings whose interference fringes are parallel to the recording material. In this way, an analytical equation for transmittance at the exit surface of the material was obtained together with an analytical expression for the fields inside the material. The comparison of such an analytical expressions with FDTD allowed us to corroborate the theoretical accurateness of our model. The analytical expression for the transmittance was fitted to the experimental transmittance data, thereby enabling various characteristic parameters of the grating to be determined including the refractive index modulation. For a thickness of around $70 \mu \mathrm{m}$, values of $\Delta n$ of around 0.003 were obtained. Throughout the study it has been shown that this is the most appropriate thickness for storing reflection gratings since lesser thicknesses give rise to lower modulations, whereas greater thicknesses prevent the recording beams from interfering adequately in the material, which also results in lower modulations. Shrinkage values were also obtained for the stored gratings, and in no case were they found to be over $2.2 \%$.

This work was supported by the "Generalitat Valenciana" (Spain) under project PROMETEO/ 2011/021, ISIC/2012/013, and GV/2012/099 and by "Ministerio de Ciencia e Innovación" (Spain) under projects FIS2011-29803-C02-01 and FIS201129803-C02-02.

\section{References}

1. L. Dhar, K. Curtis, and T. Fäcke, "Holographic data storage: coming of age," Nat. Photonics 2, 403-405 (2008).

2. D. Graham-Rowe, "The drive for holography," Nat. Photonics 1, 197-200 (2007).

3. M. Omori, H. Kondo, T. Miyata, N. Mori, H. Matsuo, T. Sasamuro, S. Okauchi, J. Ensher, R. Harris, A. Wegner, and J. Coza, "Enhancement of a tunable blue laser for holographic data storage," Proc. SPIE 7730, 77300T (2010).

4. K. Curtis, L. Dhar, A. Hill, W. Wilson, and M. Ayres, Holographic Data Storage: From Theory to Practical Systems (Wiley, 2010).

5. E. Fernandez, A. Marquez, S. Gallego, R. Fuentes, C. García, and I. Pascual, "Hybrid ternary modulation applied to multiplexing holograms in photopolymers for data page storage," J. Lightwave Technol. 28, 776-783 (2010).

6. M. Weiser, F. Bruder, T. Facke, D. Honel, D. Jurbergs, and T. Rolle, "Self-processing, diffusion-based photopolymers for holographic applications," Macromol. Symp. 296, 133-137 (2010).

7. C. Meka, R. Jallapuram, I. Naydenova, S. Martin, and V. Toal, "Development of a panchromatic acrylamide-based photopolymer for multicolor reflection holography," Appl. Opt. 49, 1400-1405 (2010).

8. I. Naydenova, R. Jallapuram, V. Toal, and S. Martin, "Characterisation of the humidity and temperature responses of a reflection hologram recorded in acrylamide-based photopolymer," Sens. Actuators B Chem. 139, 35-38 (2009).

9. R. Castagna, F. Vita, D. E. Lucchetta, L. Criante, and F. Simoni, "Superior-performance polymeric composite materials for high-density optical data storage," Adv. Mater. 21, 589-592 (2009)

10. A. Murciano, S. Blaya, L. Carretero, R. F. Madrigal, and A. Fimia, "Holographic reflection gratings in photopolymerizable solgel materials," Opt. Lett. 31, 2317-2319 (2006).

11. R. Fuentes, E. Fernandez, C. Garcia, A. Belendez, and I. Pascual, "Study of influence of ACPA in holographic reflection gratings recorded in PVA/AA based photopolymer," Proc. SPIE 7717, 77170Q (2010).

12. C. Neipp, I. Pascual, and A. Belendez, "Fixation-free rehalogenating bleached reflection holograms recorded on BB-640 plates," Opt. Commun. 182, 107-114 (2000).

13. Y. Yonetani, K. Nitta, and O. Matoba, "Numerical evaluation of angular multiplexing in reflection-type holographic data storage in photopolymer with shrinkage," Appl. Opt. 49, 694-700 (2010).

14. R. Kostuk, W. Maeda, C. Chen, I. Djordjevic, and B. Vasic, "Cascaded holographic polymer reflection grating filters for optical-code-division multiple-access applications," Appl. Opt. 44, 7581-7586 (2005).

15. L. Criante, R. Castagna, F. Vita, D. E. Lucchetta, and F. Simoni, "Nanocomposite polymeric materials for high density optical storage," J. Opt. A 11, 024011 (2009).

16. J. Zhu, G. Dong, X. Guo, L. Chen, and J. Li, "Methylene-blue sensitized dichromated gelatin: wide-range colour adjustment of reflection hologram," J. Opt. A 6, 132-136 (2004).

17. J. M. Kim, B. S. Choi, Y. S. Choi, H. I. Bjelkhagen, and N. J. Phillips, "Holographic optical elements recorded in silver halide sensitized gelatin emulsions. Part 2. Reflection 
holographic optical elements," Appl. Opt. 41, 1522-1533 (2002).

18. L. Carretero, M. Perez-Molina, S. Blaya, R. F. Madrigal, P. Acebal, and A. Fimia, "Application of the fixed point theorem for the solution of the 1D wave equation: comparison with exact Mathieu solutions," Opt. Express 13, 9078-9084 (2005).

19. C. Neipp, J. Fraces, M. Perez-Molina, S. Blenda, and A. Belendez, "Transference matrix method for non slanted holographic reflection gratings," Proc. SPIE 7717, 771706 (2010).

20. L. Carretero, M. Perez-Molina, P. Acebal, S. Blaya, and A. Fimia, "Matrix method for the study of wave propagation in one-dimensional general media," Opt. Express 14, 11385-11391 (2006).

21. M. Ortuño, S. Gallego, C. García, C. Neipp, A. Beléndez, and I. Pascual, "Optimization of a $1 \mathrm{~mm}$ thick PVA/acrylamide recording material to obtain holographic memories: method of preparation and holographic properties," Appl. Phys. B 76, 851-857 (2003).

22. R. Fuentes, E. Fernandez, C. Garcia, A. Belendez, and I. Pascual, "Study of reflection gratings recorded in polyvinyl alcohol/acrylamide-based photopolymer," Appl. Opt. 48, 6553-6557 (2009).

23. S. Gallego, C. Neipp, M. Ortuño, E. Fernández, A. Beléndez, and I. Pascual, "Analysis of multiplexed holograms stored in a thick PVA/AA photopolymer," Opt. Commun. 281, 1480-1485 (2008).

24. M. Born and E. Wolf, Principles of Optics (Macmillan, 1964).

25. M. Perez-Molina and L. Carretero, "Polynomial fixed-point algorithm applied to the electromagnetic analysis of onedimensional continuous structures," J. Opt. Soc. Am. B 24, 1354-1364 (2007).

26. I. S. Gradshteyn and I. Ryzhik, Table of Integrals, Series and Products (Academic, 1994).

27. A. Belendez, T. Belendez, C. Neipp, and I. Pascual, "Determination of the refractive index and thickness of holographic silver halide materials by use of polarized reflectances," Appl. Opt. 41, 6802-6808 (2002).

28. L. Criante, K. Beev, D. E. Lucchetta, and F. Simoni, "Spectral analysis of shrinkage in holographic materials suitable for optical storage applications," Proc. SPIE 6252, 62520G (2006). 